\title{
On the origin of Robertsonian fusions in nature: evidence of telomere shortening in wild house mice
}

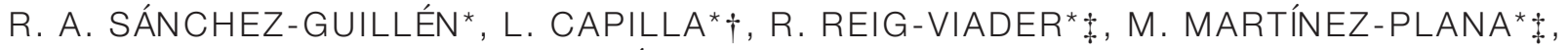 \\ C. PARDO-CAMACHO* + M. ANDRÉS-NIETO* + , J. VENTURA $+\& A$. RUIZ-HERRERA* + \\ *Genome Integrity and Instability Group, Institut de Biotecnologia i Biomedicina (IBB), Universitat Autònoma de Barcelona, Barcelona, Spain \\ $\uparrow$ Departament de Biologia Animal, Biologia Vegetal i Ecologia, Universitat Autònoma de Barcelona, Barcelona, Spain \\ $₫$ Departament de Biologia Cel lular, Fisiologia i Immunologia, Universitat Autònoma de Barcelona, Barcelona, Spain
}

Keywords:

chromosomal evolution;

Robertsonian fusions;

telomere shortening;

western house mouse.

\begin{abstract}
The role of telomere shortening to explain the occurrence of Robertsonian (Rb) fusions, as well as the importance of the average telomere length vs. the proportion of short telomeres, especially in nature populations, is largely unexplored. In this study, we have analysed telomere shortening in nine wild house mice from the Barcelona Rb system with diploid numbers ranging from 29 to 40 chromosomes. We also included two standard $(2 \mathrm{n}=40)$ laboratory mice for comparison. Our data showed that the average telomere length (considering all chromosomal arms) is influenced by both the diploid number and the origin of the mice (wild vs. laboratory). In detail, we detected that wild mice from the Rb Barcelona system (fused and standard) present shorter telomeres than standard laboratory mice. However, only wild mice with $\mathrm{Rb}$ fusions showed a high proportion of short telomeres (only in p-arms), thus revealing the importance of telomere shortening in the origin of the Rb fusions in the Barcelona system. Overall, our study confirms that the number of critically short telomeres, and not a simple reduction in the average telomere length, is more likely to lead to the origin of $\mathrm{Rb}$ fusions in the Barcelona system and ultimately in nature.
\end{abstract}

\section{Introduction}

Chromosomal rearrangements in the form of inversions, translocations and chromosome fusions/fissions are key factors for evolution. This evolutionary role is especially true for Robertsonian ( $\mathrm{Rb}$ ) fusions, whose importance is revealed by its occurrence in taxa as diverse as mammals, reptiles, insects or mollusks (White, 1973; King, 1995). For instance, Rb fusions, together with inversions, contribute to chromosomal speciation through underdominance and/or by suppression of recombination when they are present in heterozygous form (Rieseberg, 2001; Dumas \& BrittonDavidian, 2002; Faria \& Navarro, 2010; Farré et al., 2013; Capilla et al., 2014). Although most of the recent

Correspondence: Rosa A. Sanchéz-Guillén and A. Ruiz-Herrera, Genome Integrity and Instability Group, Institut de Biotecnologia i Biomedicina (IBB), Universitat Autònoma de Barcelona, Campus UAB, 08193 Barcelona, Spain.

Tel.: +34 93 5811379, +3493 5812051; fax: +3493581 3357; e-mails: rguillenuvigo@hotmail.com and aurora.ruizherrera@uab.es literature has been focused on the diversity of factors influencing the fixation of chromosomal rearrangements within populations (Faria \& Navarro, 2010 and references therein), the mechanism(s) responsible for the origin of $\mathrm{Rb}$ fusions have received less attention.

Robertsonian fusions occur when two telocentric or acrocentric chromosomes fuse resulting in one metacentric chromosome (Robertson, 1916). Several factors have been invoked for the appearance of $\mathrm{Rb}$ fusions in mammalian species; these include chromosome size, GC and DNA content, gene density, illegitimate recombination between repetitive sequences and telomere loss or inactivation (Slijepcevic, 1998; Garagna et al., 2001; Ruiz-Herrera et al., 2010; Wesche \& Robinson, 2012). Telomeres are ribonucleoprotein structures composed of TTAGGG tandem repeats, a protein complex (shelterin) and noncoding telomeric RNA (TERRA) that cap the ends of eukaryotic chromosomes to protect them from being recognized as double-strand breaks (DSBs) (Azzalin et al., 2007; O'Sullivan \& Karlseder, 2010; Reig-Viader et al., 2014). Therefore, Rb fusions 
would require either the elimination or the inactivation of telomeres prior to the chromosomal rearrangement. In this context, three different mechanisms have been formally proposed to explain the origin of $\mathrm{Rb}$ fusions in relation to the presence/absence of telomeric sequences in the resultant fused chromosome: (i) telomeric inactivation, (ii) chromosomal breakage within centromeric satellite sequences and (iii) telomeric shortening (Slijepcevic, 1998).

The first mechanism assumes the occurrence of chromosomal fusions by telomere inactivation without loss of telomeric repeats (Slijepcevic, 1998), and occasionally, the fused chromosomes would exhibit large blocks of telomeric repeats that would be retained and/or amplified in the pericentromeric regions (see Ruiz-Herrera et al., 2008 for a review). In fact, the presence of telomeres with an altered structure is common in many vertebrates (see Slijepcevic, 1998), with numerous examples of end-to-end telomere fusions in the absence of significant telomere shortening (e.g. Bailey et al., 1999; Espejel \& Blasco, 2002). According to the second mechanism (chromosomal breakage in small satellite sequences), chromosomal breaks within centromeric repetitive sequences would result in the total loss of telomeres of the p-arms, promoting fusion of the chromosomal ends. This mechanism has been proposed to explain chromosomal evolution in several rodent species (Nanda et al., 1995; Garagna et al., 2001). The last of the suggested mechanisms (telomeric shortening) proposes that $\mathrm{Rb}$ fusions could result from progressive telomere shortening due to the 'end of replication problem' (Allsopp et al., 1995). This states that due to the inefficiency of DNA polymerase to replicate the whole telomeric sequences of the lagging strand during the Sphase of mitotic division, telomeres progressively shorten, resulting in loss of the telomeric DNA and reaching critical length in the absence of telomere elongation mechanisms. This would precipitate the fusion of chromosomal ends (Slijepcevic, 1998). Knockout mice lacking telomerase in successive generations represent a good example of chromosomal fusion due to telomere shortening (Blasco et al., 1997). In fact, Hemann et al. (2001) detected a preference for the fusion of chromosomes with short telomeres in telomerase-null mice. They predict that as telomeres shorten, chromosomes undergo fusion. Nevertheless, and despite the experimental evidence, telomere length has never been formally studied in natural $\mathrm{Rb}$ populations.

The western house mouse (Mus musculus domesticus) is arguably the best studied and understood model of Rb variation in nature. The accumulation of $\mathrm{Rb}$ fusions is thought to encompass the past 10000 years (Auffray, 1993). The standard karyotype of the house mouse consists of all acrocentric chromosomes $(2 \mathrm{n}=40)$; however, a wide range of diploid numbers (2n) (from 22 to 39) has been described in the literature (Piálek et al., 2005). In fact, 106 different $\mathrm{Rb}$ fusion combinations are distributed across Europe and the Mediterranean basin. One of these $\mathrm{Rb}$ systems, the Barcelona Rb system, has diploid numbers ranging from 27 to 39 chromosomes (Medarde et al., 2012 and references therein). This system occurs in a $5000 \mathrm{~km}^{2}$ area of Barcelona province (Spain) and is characterized by a high level of chromosomal polymorphisms [i.e. seven different $\mathrm{Rb}$ chromosomes including Rb (3.8), (4.14), (5.15), (6.10), (7.17), (9.11) and (12.13) showing nongeographically coincident clines] (Medarde et al., 2012) and low recombination rates (Capilla et al., 2014). To disentangle the mechanism(s) responsible for the formation of $\mathrm{Rb}$ fusions in natural populations, we have investigated telomere shortening in the Barcelona $\mathrm{Rb}$ system. To this end, telomere length and the proportion of short telomeres were measured in standard and $\mathrm{Rb}$ mice from the system, and compared to standard laboratory mice (C57BL/6; B6). Our results indicate that telomere shortening can explain the origin of the $\mathrm{Rb}$ fusions in the Barcelona system and thus confirm the role of telomere shortening in shaping the occurrence of $\mathrm{Rb}$ fusions in nature.

\section{Materials and methods}

\section{Cell cultures and chromosome extraction}

Primary fibroblast cell lines were previously established [from nine wild male mice livetrapped in commensal habitats from five different localities representative of the Barcelona Rb system] (Fig. la) and karyotyped by Capilla et al. (2014). Cell lines were characterized by diploid numbers that ranged from 29 to $40(2 n=40$, three mice; $2 \mathrm{n}=38$, one mouse; $2 \mathrm{n}=37$, one mouse; $2 \mathrm{n}=31$, one mouse; $2 \mathrm{n}=30$, two mice; and $2 \mathrm{n}=29$, one mouse) (Table 1). In addition, two primary fibroblast cell lines were established from two laboratory mice $(2 \mathrm{n}=40, \mathrm{~B} 6)$, which together with HeLa cells (human cells with known telomere length) were included in the analysis as internal controls for telomere length measurements. Tissue culture protocols followed Capilla et al. (2014). Chromosome preparations were obtained at early passages in culture (from 2 th to 7 th).

\section{Quantitative-fluorescence in situ hybridization (Q- FISH)}

Q-FISH method on metaphases was used based on its high sensitivity, which allows the quantification of individual telomeres in single cells, as well as the identification of short telomeres (telomeric repeats $<0.15 \mathrm{kbp}$, the so-called 'signal-free ends'; Poon et al., 1999; Aubert et al., 2012), which appear as telomeres without FISH signal. Metaphase chromosomes were hybridized using the peptide-nucleic acid-FISH (PNA-FISH) preparation method described by the manufacturer 
Fig. 1 Distribution and type of $\mathrm{Rb}$ fusions. (a) Sample distribution in the Barcelona chromosomal polymorphic zone. Diploid numbers are shown for standard (green) and Rb (dark red) animals of the Rb system. (b-d) Examples of Q-FISH experiments performed on metaphases of specimens with (b) three fusions, specimen SS18, (c) standard karyotype from a B6 mouse and (d) ten fusions, specimen 968. Telomeres are shown in green (TEL) and chromosomes are stained in blue (DAPI). (a)
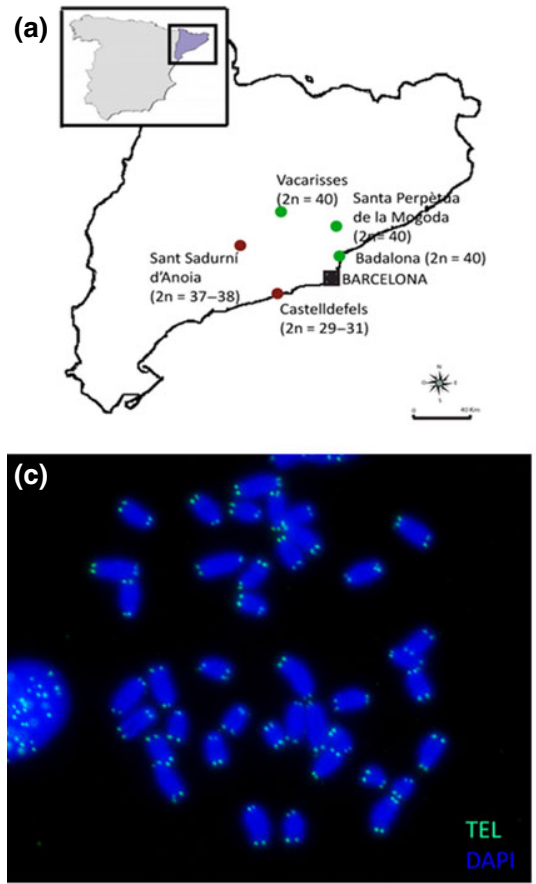

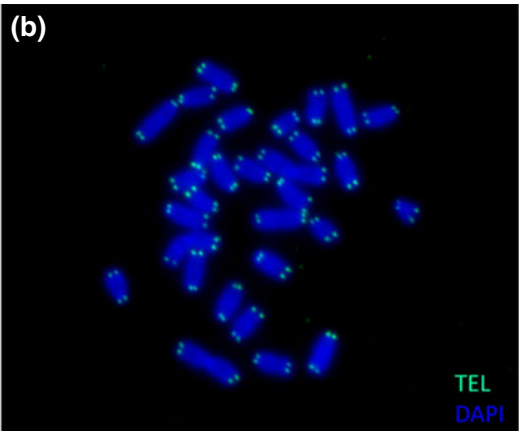

(d)

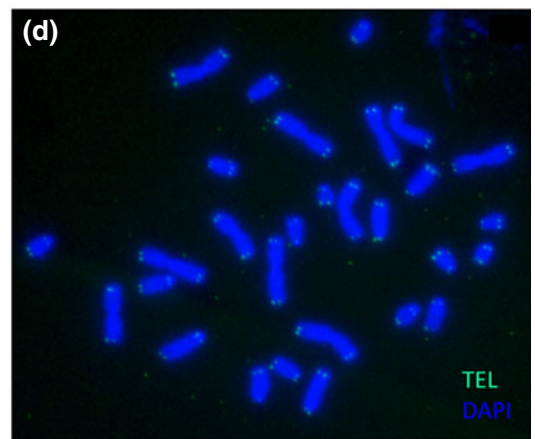

(www.panagene.com). The FAM-conjugated (CCCTAA $)_{3}$ PNA probe (telo C) (Panagen) was used to label telomeres; DNA counterstaining was performed using DAPI (40,6-diamidino-2-phenylindole). For each Q-FISH experiment, an internal control consisting of HeLa cells derived from the same chromosome extraction (i.e. same passage) was included.

\section{Telomere length measurements}

Chromosomal preparations were visualized with a Zeiss Axioskop epifluorescence microscope equipped with the appropriate filters for FAM/DAPI detection and a charged coupled device camera (ProgResR CS10plus, Jenoptik, Jena, Germany). Images were captured and produced by the PROGRESR software (2.7.7), maintaining the same exposure time. Fluorescence intensities of telomeres were analysed with the TFLTELO-v2 software package (Terry Fox Laboratory, BC Cancer Research Centre, Vancouver, BC, Canada) (Poon et al., 1999). Analyses of telomere fluorescence were carried out maintaining the same tolerance threshold values. At least 28 metaphases were analysed per cell line (Table 1) given that this sample size gives comparable results to conventional southern analysis (Poon et al., 1999; Vera \& Blasco, 2012). Telomere lengths were obtained in terms of arbitrary telomere fluorescence units (TFUs) following previous studies (Reig-Viader et al., 2014).

We investigated the phenomenon of telomere shortening in terms of telomere length and proportion of short telomeres, which normally participate in end- to-end fusions. To this end, q- and p-arms of each acrocentric and metacentric (fused) chromosome were identified in each metaphase and their telomere lengths and proportion of short telomeres were measured. In this study, telomeres of metacentric chromosomes were always considered as q-arm telomeres. Analyses were performed following three approaches. Firstly, we considered only the type of chromosome arm (q-arms vs. p-arms). Secondly, we considered only diploid numbers, and thus we included six groups: (i) $2 \mathrm{n}=40$, (ii) $2 \mathrm{n}=38$, (ii) $2 \mathrm{n}=37$, (iv) $2 \mathrm{n}=31$, (v) $2 \mathrm{n}=30$ and (vi) $2 \mathrm{n}=29$. Thirdly, analyses were performed considering both origin and type of mice: (i) Laboratory-standard, which included B6 laboratory mice ( $2 \mathrm{n}=40$, B6, two mice); (ii) Rb-standard, which included standard wild mice from the $\mathrm{Rb}$ system ( $2 \mathrm{n}=40$, three mice) and (iii) Rb-fused, which included wild mice from the Rb system with $\mathrm{Rb}$ fusions ( $2 \mathrm{n}=29$ to $2 \mathrm{n}=38$, six mice).

\section{Correlation between $\mathrm{q}$ - and p-arm telomeres}

Telomere length data were normally distributed, and thus we used an ANova (as implemented in JMP 1lth version) to compare q-and p-arm telomere lengths and also to test whether $\mathrm{p}$-arms are more prone to loss telomere FISH signals (Almeida \& Ferreira, 2013). Additionally, we investigated whether telomere shortening is similarly affecting q- and p-arms. To this end, length ratio $(\mathrm{q}-\mathrm{arm} / \mathrm{p}-\mathrm{arm})$ in the three types of mice was investigated using an ANOvA. 


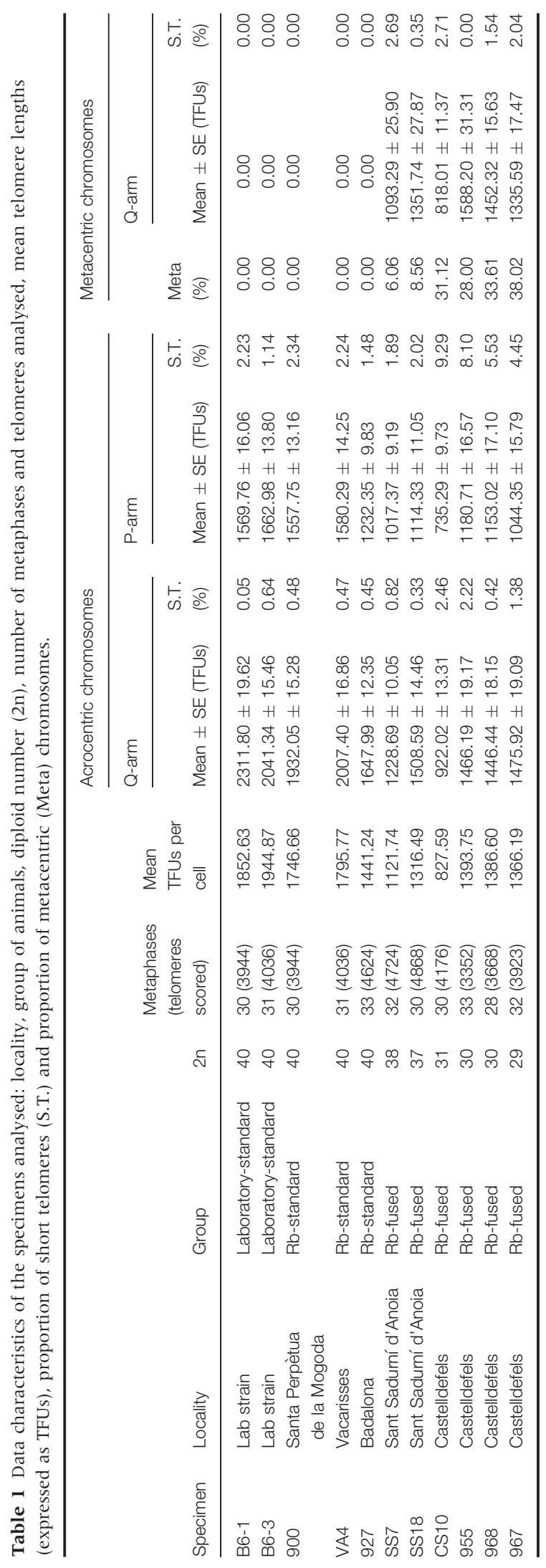

\section{Telomere shortening: average telomere length and proportion of short telomeres}

The relationship between telomere length and: (i) type of chromosomal arm (q- and p-arms), (ii) diploid number (from $2 \mathrm{n}=29$ to $2 \mathrm{n}=40$ ) and (iii) type of mice (Laboratory-standard, Rb-standard and Rb-fused) was examined using general linear models (GLMs) as implemented in JMP lith version. Telomere length (expressed as TFUs) was analysed with a GLM using a normal distribution and an identity-link function, with telomere length (TFUs) as the response variable and the type of arm, diploid number and the group of animals as predictor variables. The significance of parameters was determined from the Wald chi-square statistic. Additionally, the relationship between the proportion of short telomeres and: (i) type of chromosomal arm, (ii) diploid number and (iii) the type of mice was analysed using a GLM with a binomial distribution (assigning 1 for telomeres without signal telomeres and 0 for telomeres with signal) and a logit-link function with the number of short telomeres as the response variable, and the type of chromosomal arm, diploid number and group of animals as predictors.

ANOva test (telomere length) and Kruskal-Wallis (short telomeres) and subsequent post hoc analysis were applied to investigate response variables (type of chromosomal arm and group of animals) with a significant effect over telomere length and/or over the proportion of short telomeres. Additionally, the correlation (Pearson's) between diploid number and telomere length and/or proportion of short telomeres was tested by a post hoc linear regression with telomere length or with the proportion of short telomeres as the response variable and the diploid number as the predictor variable.

\section{Results}

\section{Data description}

We studied the effect of telomere shortening in the occurrence of $\mathrm{Rb}$ fusions in nature by analysing a total of 44295 telomeres including 11 mice (28-33 metaphases of each cell line; Fig. 1, Table 1). Telomere lengths followed a normal distribution in all cell lines, including HeLa control cell line (Fig. 2).

Telomere length (TFUs) of the laboratory mice (Laboratory-standard) (mean $\pm \mathrm{SE}$ per population $=$ $1898 \pm 8.83$ TFUs; $n=7899$ telomeres) ranged from 91.81 to 5806.23 TFUs, of the standard mice from the $\mathrm{Rb}$ system (Rb-standard) (mean $\pm \mathrm{SE}$ per population $=1658 \pm 6.02$ TFUs; $n=14040$ telomeres) ranged from 104.4 to 12383 TFUs and of the $\mathrm{Rb}$ mice from the $\mathrm{Rb}$ system (Rb-fused) (mean $\pm \mathrm{SE}$ per population $=1216 \pm 3.9$ TFUs; $n=21800$ telomeres) ranged from 18.0 to 9383.23 TFUs. Table 1 summarizes all data 
Fig. 2 Telomere length distribution. Distribution of telomeres for each measured TFUs in the specimens analysed. Lab strain (individuals from laboratory strain) (dark blue), $\mathrm{Rb}$ standard (individuals from the $\mathrm{Rb}$ population with standard karyotype) (green), Rb-fused (individuals from the $\mathrm{Rb}$ population with $\mathrm{Rb}$ fusions) (dark red) and HeLa (grey) lines.

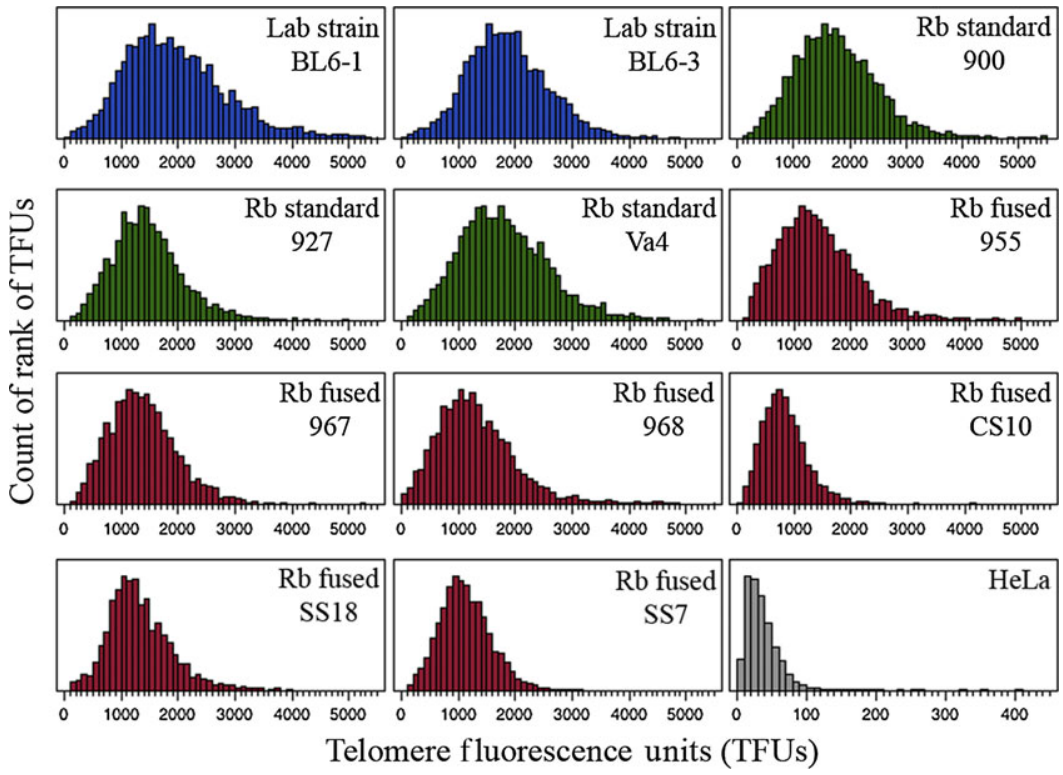

(telomeres scored, mean and SE (TFUs), percentage of short arms and percentage of metacentric chromosomes) per specimen and type of chromosomal arm. We found that mouse cells showed wider telomere length ranges and higher mean telomeric lengths than human (HeLa) cells confirming previous observations (Zijlmans et al., 1997; Reig-Viader et al., 2014).

\section{Correlation between q- and p-arm telomeres}

It is known that, in wild-type mice, p-arm telomeres are significantly shorter than q-arm telomeres (see Zijlmans et al., 1997). Our analysis confirmed this trend given that telomere lengths were significantly different between q- and p-arms (ANOVA: $F=1697 ; P<0.0001$ ). Our analysis confirmed that acrocentric p-arm telomeres are significantly shorter (mean \pm SD: $1310.93 \pm 627.85)$ than their q-arm counterparts (1699.27 \pm 775.05) $(t=54.46, P<0.0001$; Fig. 3a) and significantly similar to the telomere length of q-arms of metacentric chromosomes $(1312.93 \pm 747.24)(t=1.02$, $P=0.84$; Fig. 3a). However, acrocentric q-arms have significantly higher telomere lengths than metacentric q-arms $(t=37.58, P<0.0001$; Fig. 3a).

Thus, if $p$-arm telomeres are significantly shorter than q-arm telomeres, they may be more sensitive to lose telomeric repeats than q-arm telomeres (Blasco et al., 1997). According to this view, the proportion of short telomeres was significantly different between q- and p-arms of acrocentric and metacentric chromosomes $\left(\chi^{2}=286.64, P<0.0001\right.$; Fig. $\left.3 b\right)$. P-arms of acrocentric chromosomes have a higher proportion of short telomeres $(3.16 \%)$ than q-arms of the metacentrics $(2.07 \%)$ (Fig. $3 \mathrm{~b})$, and both, have higher proportion of short telomeres than q-arms of the acrocentric chromosomes $(0.77 \%)$ (Fig. 3b).

Q-arm/p-arm telomere length ratio among type of mice (Laboratory-standard, Rb-standard and Rb-fused) was also significantly different (ANova: $F=14.31$, $P<0.0001)$. This ratio was significantly lower in the standard mice from the Rb system (mean $\pm \mathrm{SD}$ : $1.50 \pm 0.94)(t=4.74, P<0.0001)$ than both laboratory mice $(1.62 \pm 1.30)$ and $\mathrm{Rb}$ mice from the Rb system $(1.59 \pm 01.61) \quad(t=4.29, \quad P<0.0001)$, but not significantly different between laboratory and $\mathrm{Rb}$ mice $(t=1.24, P=0.21)$. The decrease of the $q$-arm/p-arm telomere length ratio in the standard mice from the $\mathrm{Rb}$ system is consistent with a significant correlation $(r=0.156, P<0.001)$ between $\mathrm{q}-$ and $\mathrm{p}$-arms, in both laboratory mice $(r=0.114, P<0.0001)$ and $\mathrm{Rb}$ mice from the Rb system $(r=0.108, P<0.0001)$.

\section{Telomere shortening: average telomere length and proportion of short telomeres}

To examine the role of telomeric shortening on the origin of the $\mathrm{Rb}$ fusions in the Barcelona $\mathrm{Rb}$ system, telomere length and proportion of short telomeres were analysed with GLMs. Table 2 includes Wald chi-square values, degrees of freedom and $P$ values.

Overall, we observed that average telomere length was influenced by the type of chromosomal arm (q and $\mathrm{p}$ ), the group of mice (Laboratory-standard, Rbstandard and $\mathrm{Rb}$-fused) and the diploid number (from $2 \mathrm{n}=29$ to $2 \mathrm{n}=40$ ) (Table 2 ). In Rb-fused mice, the average telomere length (mean $\pm \mathrm{SD}) \quad(\mathrm{q}$-arm $=$ $1333.63 \pm 604.24$ and $\mathrm{p}$-arm $=1036.32 \pm 488.40)$ was significantly shorter than both, the average telomere 

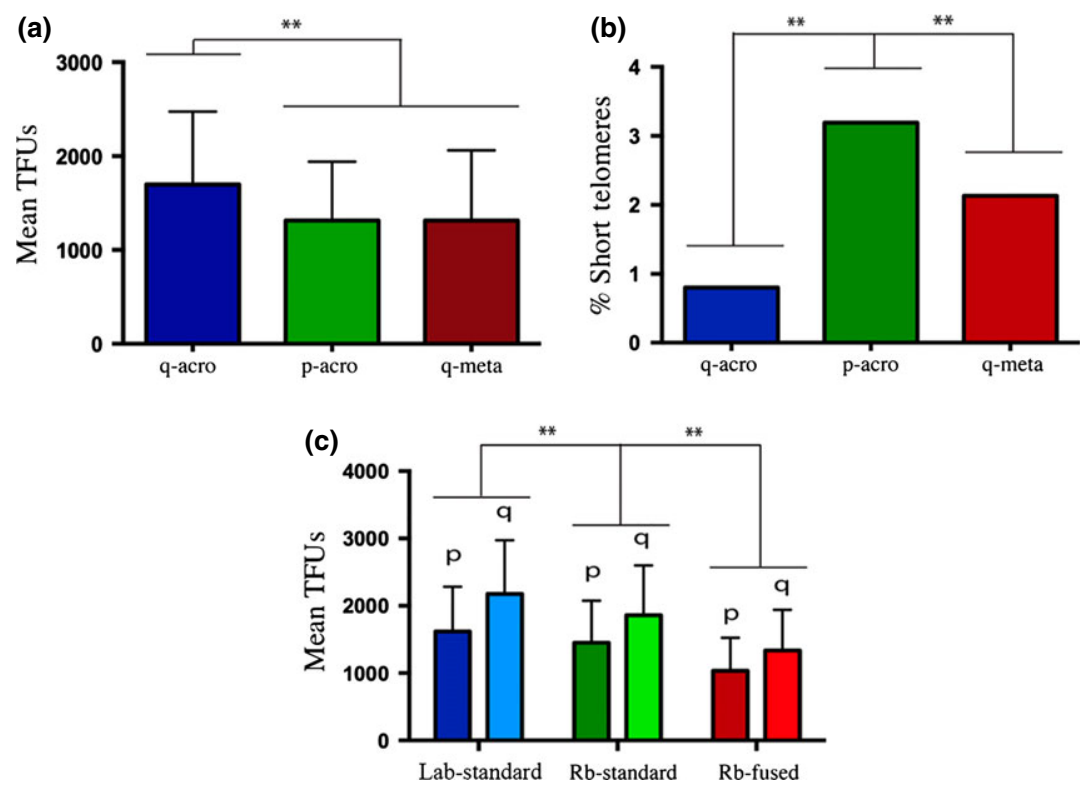

Fig. 3 Telomere shortening analysis. (a) Mean telomere lengths (in TFUs) for each type of chromosomal arms: p-arms of acrocentric chromosomes (p-acro), q-arms of acrocentric chromosomes (q-acro) and q-arms of metacentric chromosomes (q-meta) (Student's t-test, ** $P$-value $<0.001$ ). (b) Percentage of short telomeres for each type of chromosomal arms: p-arms of acrocentric chromosomes (p-acro), qarms of acrocentric chromosomes (q-acro) and q-arms of metacentric chromosomes (q-meta) (Student's $t$-test, $* * P$-value $<0.001$ ). (c) Mean telomere lengths (in TFUs) for each type of chromosomal arms (p- and q-arms) for the three different animal groups compared: laboratory mice (Lab-standard), standard mice from the Rb system (Rb-standard) and Rb mice from the Rb system (Rb-fused) (Student's $t$ test, ${ }^{* *} P$-value $\left.<0.001\right)$.

Table 2 Generalized linear model for telomere length and proportion of short telomeres according to diploid number $(2 \mathrm{n})$, group of animals (Laboratory-standard, Rb-Standard and Rb-Fused) and chromosome arm (q-and p-arms).

\begin{tabular}{|c|c|c|c|c|c|c|}
\hline \multirow[b]{2}{*}{ Fixed effects } & \multicolumn{3}{|c|}{ Telomere length } & \multicolumn{3}{|c|}{ Short telomeres } \\
\hline & $\chi^{2}$ & d.f. & $P$ & $\chi^{2}$ & d.f. & $P$ \\
\hline $2 \mathrm{n}$ & 11.97 & 1 & $0.0005 *$ & 113.17 & 1 & $<0.0001 *$ \\
\hline Group & 3390.67 & 2 & $<0.0001 *$ & 2.44 & 2 & 0.2953 \\
\hline Arm & 3351.69 & 2 & $<0.0001 *$ & 353.00 & 2 & $<0.0001^{*}$ \\
\hline
\end{tabular}

$P$ refers to type II deviance values. * indicates statistical significance.

length of the Rb-standard mice $(\mathrm{q}$-arm $=1859.80$ \pm 739.83 and $\mathrm{p}$-arm $=1453.19 \pm 622.64)$ (q-arm: $t=46.55, \quad P<0.0001 ;$ p-arm: $t=43.90, \quad P<0.0001$; Fig. 3c) and the Laboratory-standard mice (qarm $=2175.40 \pm 797.26$ and p-arm $=1617.17 \pm 662.99$ ) (q-arm: $\quad t=62.49, \quad P<0.0001 ; \quad \mathrm{p}$-arm: $t=51.44$, $P<0.0001$; Fig. 3c). Moreover, average telomere length of Rb-standard mice was significantly shorter than average telomere length of the Laboratory-standard mice (q-arm: $t=22.80, P<0.0001 ; p$-arm: $t=14.18$, $P<0.0001$; Fig. 3c). Finally, and only in the case of Rb-fused mice, due to their unique composition of metacentric and acrocentric q-arms, when comparing average telomere length of acrocentric $(1333.63 \pm 604.24)$ and metacentric chromosomes in $\mathrm{Rb}$ mice $(1300.05 \pm 747.44)$, we detected that metacentric q-arms were significantly shorter $(t=3.38$, $P=0.0007)$.

Moreover, diploid numbers correlated significantly with telomere length of both type of chromosomal arms (q-arm: $r=0.103, P<0.0001$; p-arm: $r=0.092$, $P<0.0001)$, that is, shorter average telomeres appeared in mice with lower diploid numbers (with more $\mathrm{Rb}$ fusions) (Fig. 4a,b). However, only telomere length of q-arms correlated with number of fusions (q-arm: $r=0.0006, P=0.02$; p-arm: $r=0.0001, P=0.35$ ).

Conversely, the proportion of short telomeres was influenced by both, the type of chromosomal arm and the diploid number but not by the type of mice (see Table 2). The percentage of short telomeres in both chromosomal arms (q- and p-arms) was significantly correlated with the diploid number (q-arm: $r=0.003$, $P<0.0001$; p-arm: $r=0.013, \quad P<0.0001$; Fig. 4c,d) and also with the number of fusions (q-arm: $r=0.002$, $P<0.0001$; p-arm: $r=0.011, P<0.0001)$.

\section{Discussion}

The role of telomere shortening to explain $\mathrm{Rb}$ fusions in natural populations, as well as the importance of the average telomere length vs. the proportion of short 


\author{
Fig. 4 Karyotype and telomere length. \\ Correlation analysis between diploid \\ number and mean telomere length \\ (expressed as TFUs) for each type of \\ chromosomal arms: (a) q-arms and (b) \\ p-arms. Correlation analysis between \\ diploid numbers expressed percentage \\ of short telomeres of (c) q-arms and (d) \\ p-arms.
}
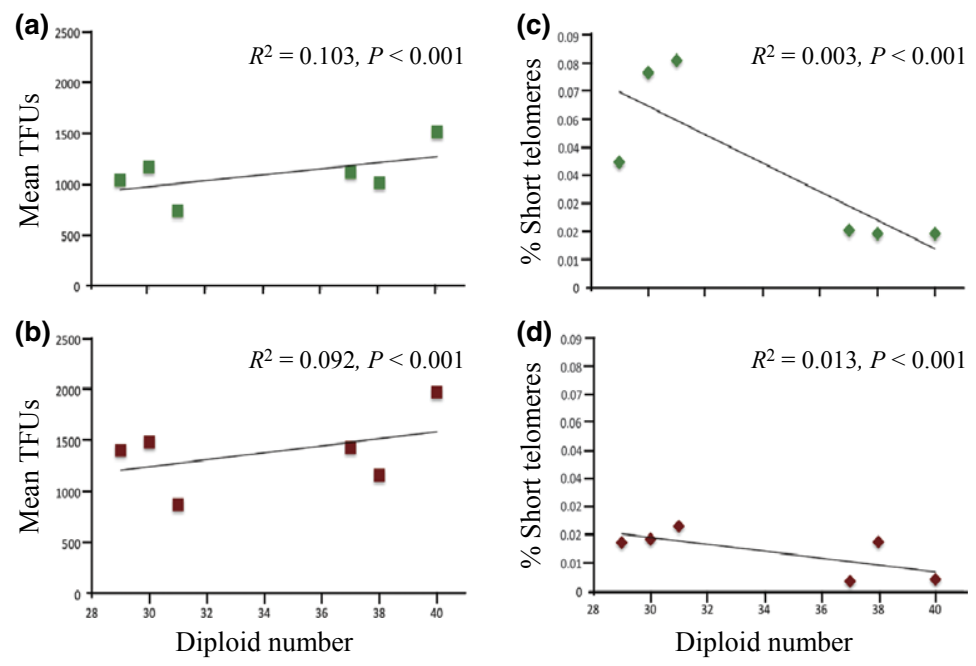

telomeres is yet under discussion. It is known that telomere length can vary among species, individuals and even among telomeres within the same cell (Zijlmans et al., 1997; Canela et al., 2007). As the generation of $\mathrm{Rb}$ fusions due to telomere shortening has been observed exclusively in vitro (i.e. in tissue cultures established from telomerase-null mice) (Blasco et al., 1997), the occurrence of such mechanisms in nature, together with the stabilization of the resultant chromosomes through subsequent generations, needs further validation. Here, we provide the first evidence of telomere shortening, in terms of telomere length and percentage of short telomeres, in a wild house mouse $\mathrm{Rb}$ system. We detected that both types of wild mice from the Barcelona $\mathrm{Rb}$ system (with $\mathrm{Rb}$ fusions and standards) presented, on average, significantly lower telomere lengths than laboratory standard mice, and this correlation held when considering p- and q-arms. Additionally, and more interestingly, the percentage of short telomeres was (in this case, only for the q-arms) negatively correlated to the diploid number, but was influenced by the type of mice, because only mice with $\mathrm{Rb}$ fusions showed high proportions of short telomeres.

It is well established that mouse p-arm telomeres are shorter than their q-arm counterparts (Zijlmans et al., 1997). We found that the length of telomeres on qand p-arms differed among the three groups of mice analysed and that this was influenced by the diploid number. In fact, in wild mice from the Barcelona $\mathrm{Rb}$ system, p-arm telomeres were significantly shorter than their q-arm counterparts and presented a higher proportion of short telomeres than their q-arm counterparts. Additionally, and consistently with Nanda et al. (1995) study on laboratory mice strains with Rb fusions, we did not detect telomeric signals at the centromeres of the metacentric chromosomes in the analysed specimens. Moreover, we observed that the average telomere length of the metacentric chromosomes was more similar (although significantly higher) to the average length of the q-arms telomeres of the acrocentric chromosomes, than to the average length of their p-arm telomeres. Therefore, these results indicate that $\mathrm{Rb}$ fusion formation between $\mathrm{p}$-arms would most probably be triggered by the loss of whole p-arm telomeres.

Previous studies on telomerase-null mice (Blasco et al., 1997; Hemann et al., 2001) showed that chromosomes with short telomeres have a preference for fusion, and based on this finding, they predict that as telomeres shorten, chromosomes would undergo fusions as a way to stabilize chromosomal ends. Consistent with this prediction, we have similarly detected that wild mice with $\mathrm{Rb}$ fusions have shorter telomeres (q- and p-arms) than standard mice (from the Rb system and laboratory mice), and the diploid number was negatively correlated with the proportion of short telomeres. Therefore, considering the three different mechanisms that have been proposed to explain the origin of $\mathrm{Rb}$ fusions in relation to the presence/absence of telomeric repeats in the resultant chromosome, we suggest that telomere shortening is involved in the formation of $\mathrm{Rb}$ fusions in natural populations. Known as the widespread distribution of $\mathrm{Rb}$ fusions in the Barcelona polymorphic zone (Medarde et al., 2012 and references therein), Rb fusions would represent an effective way to stabilize the progressive loss of telomeric sequences within the population.

The fact that mice from the Barcelona Rb system, and especially those with $\mathrm{Rb}$ fusions, present shorter telomeres when compared to standard laboratory mice is an intriguing result and highlights the importance of focusing on natural populations. Some studies have suggested that telomere length is under genetic control, for example humans (Slagboom et al., 1994; Jeanclos et al., 2000; Vasa-Nicotera et al., 2005) and mice (Zhu et al., 1998; Ding et al., 2004). Zhu et al. (1998) showed, based on the telomere length polymorphism 
between mice of the interfertile species Mus spretus and M. musculus, that an unidentified gene located in a region on distal chromosome 2 regulates telomere length in mice. More recently, Ding et al. (2004) identified Rtel (a 'regulator of the telomere gene') that is thought to codify a helicase which is implicated in telomere length regulation and prevents genetic instability. We detected that standard mice from the $\mathrm{Rb}$ system had shorter telomere lengths in both q-and p-arms but similar proportions of short telomeres than the standard mice from the laboratory strain. The low average length of both types of wild mice from the Barcelona Rb systems (standard and fused) could be explained by the presence and/or dominance of an allelic variant of gene (s) coding for protein(s) involved in telomere length maintenance. In this way, chromosome fusions would take place when telomere length reached the threshold recognized by the DSBs repair machinery.

Apart from telomerase activity, the so-called alternative lengthening of telomeres (ALT) has been reported as an additional telomerase-independent mechanism involved in telomere lengthening and maintenance through homologous recombination (HR) between telomeres (Cesare \& Reddel, 2010). Although this mechanism has been mainly described in tumour cell lines (Bryan et al., 1995; Dunham et al., 2000), it has been reported that short telomeres have an increased level of telomere recombination in primary cells (i.e. in the absence of telomerase), suggesting that the ALT mechanism can be present as a way to stabilize telomeric-free ends (Neumann et al., 2013). Recently, we have reported a displacement of recombination events to telomeres in fused metacentric chromosomes of spermatocytes of $\mathrm{Rb}$ wild mice from the Barcelona Rb system (Capilla et al., 2014). Such observation, together with the fact that telomeres are significantly shorter in wild mice (standard and fused) from the Barcelona Rb system compared to standard laboratory mice, suggests that the HR could be contributing to telomere length maintenance in the Barcelona $\mathrm{Rb}$ system, maybe by the ALT mechanism. Therefore, telomeres would be maintained to avoid telomeric lengths below $15 \mathrm{kbp}$ (which would be close to the critic length that leads telomeres to be recognized as DSBs by the repair machinery). In this way, cells avoid fusions between all acrocentric chromosomes in Rb mice and would delay the generation of fused metacentric chromosomes in standard mice.

In conclusion, we detected that Rb-fused mice present shorter $\mathrm{q}$ - and $\mathrm{p}$-arms telomeres and higher percentage of short telomeres (only in p-arms) than the standard mice. However, standard mice from the Barcelona $\mathrm{Rb}$ system showed, on average, shorter telomeres, but similar proportion of short telomeres than the standard mice from the B6 laboratory strain. This fact suggests that the number of critically short telomeres, and not the reduction in the telomere length per se, is probably the responsible of the occurrence of $\mathrm{Rb}$ fusions in wild mice from the Barcelona Rb system.

\section{Acknowledgments}

The authors acknowledge T. J. Robinson for insightful comments on earlier versions of the manuscript and to Francisca Garcia and Francisco Cortés from the SCAC (Servei de Cultius Cel.lulars, Universitat Autónoma de Barcelona) for their assistance with the cell lines. We are also grateful to Nuria Medarde for her contribution in the fieldwork. RAS-G was supported by a postdoctoral grant from 'Alianza 4 Universidades', LC is the beneficiary of a FPI predoctoral fellowship (BES-2011-047722), and RRV was supported by a Personal Investigador en Formació (PIF) fellowship of the Universitat Autònoma de Barcelona. This study was partially supported by a grant from the Ministerio de Economia y Competitividad (MINECO, CGL-2010-20170) to ARH. The capture of mice in the wild was supported by a grant from the Ministerio de Economia y Competitividad (MINECO, CGL2010-15243) to JV.

\section{References}

Allsopp, R.C., Chang, E., Kashefi-Aazam, M., Rogaev, E., Piatyszek, M.A., Shay, J.W. et al. 1995. Telomere shortening is associated with cell division in vitro and in vivo. Exp. Cell Res. 220: 194-200.

Almeida, H. \& Ferreira, M.G. 2013. Spontaneous telomere to telomere fusions occur in unperturbed fission yeast cells. Nucleic Acids Res. 41: 3056-3067.

Aubert, G., Hills, M. \& Lansdorp, P.M. 2012. Telomere length measurement-caveats and a critical assessment of the available technologies and tools. Mutat. Res. 730: 59-67.

Auffray, J.-C. 1993. Chromosomal divergence in house mice in the light of palaeontology: a colonization-related event? Quatern. Int. 19: 21-25.

Azzalin, C.M., Reichenbach, P., Khoriauli, L., Giulotto, E. \& Lingner, J. 2007. Telomeric repeat containing RNA and RNA surveillance factors at mammalian chromosome ends. Science 318: 798-801.

Bailey, S.M., Meyne, J., Chen, D.J., Kurimasa, A., Li, G.C., Lehnert, B.E. et al. 1999. DNA double-strand break repair proteins are required to cap the ends of mammalian chromosomes. Proc. Natl. Acad. Sci. U.S.A. 96: 14899-14904.

Blasco, M.A., Lee, H.W., Hande, M.P., Samper, E., Lansdorp, P.M., DePinho, R.A. et al. 1997. Telomere shortening and tumor formation by mouse cells lacking telomerase. Cell $\mathbf{9 1}$ : $25-34$.

Bryan, T.M., Englezou, A., Gupta, J.Y., Bacchetti, S. \& Reddel, R.R. 1995. Telomere elongation in immortal human cells without detectable telomerase activity. ЕMBO J. 14: 4240.

Canela, A., Vera, E., Klatt, P. \& Blasco, M.A. 2007. High-throughput telomere length quantification by FISH and its application to human population studies. Proc. Natl. Acad. Sci. U.S.A. 104: 5300-5305.

Capilla, L., Medarde, N., Alemany-Schmidt, A., Oliver-Bonet, M., Ventura, J. \& Ruiz-Herrera, A. 2014. Genetic 
recombination variation in wild Robertsonian mice: on the role of chromosomal fusions and Prdm9 allelic background. Proc. R. Soc. B 281: 20140297.

Cesare, A.J. \& Reddel, R.R. 2010. Alternative lengthening of telomeres: models, mechanisms and implications. Nat. Rev. Genet. 11: 319-330.

Ding, H., Schertzer, M., Wu, X., Gertsenstein, M., Selig, S., Kammori, M. et al. 2004. Regulation of murine telomere length by Rtel: an essential gene encoding a helicase-like protein. Cell 117: 873-886.

Dumas, D. \& Britton-Davidian, J. 2002. Chromosomal rearrangements and evolution of recombination: comparison of chiasma distribution patterns in standard and Robertsonian populations of the house mouse. Genetics 162: 1355-1366.

Dunham, M.A., Neumann, A.A., Fasching, C.L. \& Reddel, R.R. 2000. Telomere maintenance by recombination in human cells. Nat. Genet. 26: 447-450.

Espejel, S. \& Blasco, M.A. 2002. Identification of telomere-dependent "senescence-like" arrest in mouse embryonic fibroblasts. Exp. Cell Res. 276: 242-248.

Faria, R. \& Navarro, A. 2010. Chromosomal speciation revisited: rearranging theory with pieces of evidence. Trends Ecol. Evol. 25: 660-669.

Farré, M., Micheletti, D. \& Ruiz-Herrera, A. 2013. Recombination rates and genomic shuffling in human and chimpanzee-a new twist in the chromosomal speciation theory. Mol. Biol. Evol. 30: 853-864.

Garagna, S., Marziliano, N., Zuccotti, M., Searle, J.B., Capanna, E. \& Redi, C.A. 2001. Pericentromeric organization at the fusion point of mouse Robertsonian translocation chromosomes. Proc. Natl. Acad. Sci. U.S.A. 98: 171-175.

Hemann, M.T., Strong, M.A., Hao, L.Y. \& Greider, C.W. 2001. The shortest telomere, not average telomere length, is critical for cell viability and chromosome stability. Cell 107: 67-77.

Jeanclos, E., Schork, N.J., Kyvik, K.O., Kimura, M., Skurnick, J.H. \& Aviv, A. 2000. Telomere length inversely correlates with pulse pressure and is highly familial. Hypertension 36: 195-200.

King, M. 1995. Species evolution: the role of chromosome change. Syst. Biol. 44: 578 .

Medarde, N., López-Fuster, M.J., Muñoz-Muñoz, F. \& Ventura, J. 2012. Spatio-temporal variation in the structure of a chromosomal polymorphism zone in the house mouse. Heredity 109: 78-89.

Nanda, I., Schneider-Rasp, S., Winking, H. \& Schmid, M. 1995. Loss of telomeric sites in the chromosomes of Mus musculus domesticus (Rodentia: Muridae) during Robertsonian rearrangements. Chromosome Res. 3: 399-409.

Neumann, A.A., Watson, C.M., Noble, J.R., Pickett, H.A., Tam, P.P. \& Reddel, R.R. 2013. Alternative lengthening of telomeres in normal mammalian somatic cells. Genes Dev. 27: $18-23$.

O'Sullivan, R.J. \& Karlseder, J. 2010. Telomeres: protecting chromosomes against genome instability. Nat. Rev. Mol. Cell Biol. 11: 171-181.
Piálek, J., Hauffe, H.C. \& Searle, J.B. 2005. Chromosomal variation in the house mouse. Biol. J. Linn. Soc. 84: 535-563.

Poon, S.S., Martens, U.M., Ward, R.K. \& Lansdorp, P.M. 1999. Telomere length measurements using digital fluorescence microscopy. Cytometry 36: 267-278.

Reig-Viader, R., Vila-Cejudo, M., Vitelli, V., Buscà, R., Sabaté, M., Giulotto, E. et al. 2014. Telomeric repeat-containing RNA (TERRA) and telomerase are components of telomeres during mammalian gametogenesis. Biol. Reprod. 90: 103.

Rieseberg, L.H. 2001. Chromosomal rearrangements and speciation. Trends Ecol. Evol. 16: 351-358.

Robertson, W. 1916. Chromosome studies. I. Taxonomic relationships shown in the chromosomes of Tettigidae and Acrididae. V-shaped chromosomes and their significance in Acrididae, Locustidae and Gryllidae: chromosome and variation. J. Morphol. 27: 179-331.

Ruiz-Herrera, A., Nergadze, S.G., Santagostino, M. \& Giulotto, E. 2008. Telomeric repeats far from the ends: mechanisms of origin and role in evolution. Cytogenet. Genome Res. 122: 219228.

Ruiz-Herrera, A., Farré, M., Ponsà, M. \& Robinson, T.J. 2010. Selection against Robertsonian fusions involving housekeeping genes in the house mouse: integrating data from gene expression arrays and chromosome evolution. Chromosome Res. 18: 801-808.

Slagboom, P.E., Droog, S. \& Boomsma, D.I. 1994. Genetic determination of telomere size in humans: a twin study of three age groups. Am. J. Hum. Genet. 55: 876-882.

Slijepcevic, P. 1998. Telomeres and mechanisms of Robertsonian fusion. Chromosoma 107: 136-140.

Vasa-Nicotera, M., Brouilette, S., Mangino, M., Thompson, J.R., Braund, P., Clemitson, J.R. et al. 2005. Mapping of a major locus that determines telomere length in humans. Am. J. Hum. Genet. 76: 147-151.

Vera, E. \& Blasco, M. 2012. Beyond average: potential for measurement of short telomeres. Aging 4: 379-392.

Wesche, P.L. \& Robinson, T.J. 2012. Different patterns of Robertsonian fusion pairing in Bovidae and the house mouse: the relationship between chromosome size and nuclear territories. Genet. Res. 94: 97-111.

White, M.J.D. (1973). Animal Cytology and Evolution, 3rd edn (N.). Cambridge University Press, Cambridge.

Zhu, L., Hathcock, K.S., Hande, P., Lansdorp, P.M., Seldin, M.F. \& Hodes, R.J. 1998. Telomere length regulation in mice is linked to a novel chromosome locus. Proc. Natl. Acad. Sci. U.S.A. 95: 8648-8653.

Zijlmans, J.M., Martens, U.M., Poon, S.S., Raap, A.K., Tanke, H.J., Ward, R.K. et al. 1997. Telomeres in the mouse have large inter-chromosomal variations in the number of T2AG3 repeats. Proc. Natl. Acad. Sci. U.S.A. 94: 7423-7428.

Received 6 October 2014; revised 21 November 2014; accepted 23 November 2014 